\title{
Familial Creutzfeldt-Jakob Disease with a V180I Mutation: Comparative Analysis with Pathological Findings and Diffusion-Weighted Images
}

\author{
Kazuo Mutsukura ${ }^{a}$ Katsuya Satoh ${ }^{a}$ Susumu Shirabe ${ }^{c}$ Itsuro Tomita $^{d}$ \\ Takayasu Fukutome $^{\mathrm{e}}$ Minoru Morikawa ${ }^{b}$ Masachika Iseki ${ }^{f} \quad$ Kensuke Sasakig $^{g}$ \\ Yusei Shiagah $^{\text {h }}$ Tetsuyuki Kitamoto $^{i}$ Katsumi Eguchi ${ }^{a}$ \\ ${ }^{a}$ First Department of Internal Medicine and ${ }^{b}$ Department of Radiology and Radiation Biology, Graduate School \\ of Biomedical Sciences, Nagasaki University, and ' Organization of Rural Medicine and Residency Education, \\ Nagasaki University Hospital, Nagasaki, d Nagasaki Kita Hospital, Togitsu, e Kawatana National Hospital, Kawatana, \\ fPathology, Sasebo Kyosai Hospital, Sasebo, ${ }^{9}$ Department of Neuropathology, Neurological Institute, \\ Graduate School of Medical Sciences, Kyushu University, Fukuoka, and h Aoba Neurosurgery and \\ iDepartment of Neurological Science, Graduate Medical School of Tohoku University, Sendai, Japan
}

\section{Key Words}

Creutzfeldt-Jakob disease • Diffusion-weighted imaging • Magnetic resonance spectroscopy $\cdot$ Single-photon emission computed tomography • Brain biopsy

\begin{abstract}
Background: Diffusion-weighted imaging (DWI) has been reported to be a useful technique for diagnosing CreutzfeldtJakob disease (CJD). The present study reported DWI results in cases of familial CJD with a V180I mutation (CJD180) in the prion protein gene as well as neurological findings. Methods: A retrospective analysis of 3 patients with $\mathrm{V} 180 \mathrm{l}$ was performed. Cerebrospinal fluid (CSF) analysis, brain MRI, single-photon emission computed tomography (SPECT), and magnetic resonance spectroscopy (MRS) were included. CSF was analyzed for biochemical markers, and each patient underwent brain MRI, SPECT, and MRS analysis. A brain biopsy from the frontal cortex, which corresponded to the area of increased DWI signals, was utilized for neuropathological analysis. Results: CSF analysis results revealed elevated total
\end{abstract}

tau protein and the absence of 14-3-3 protein, as well as decreased concentrations of neuron-specific enolase, S100 protein, and prostaglandin $E_{2}$. All patients presented with unique MRI features. Brain biopsy showed severe spongiform morphology, but comparatively preserved neurons and mild astrocytic gliosis. Accumulations of $\mathrm{PrPSc}^{\mathrm{Sc}}$ were not detected using the 3F4 antibody, and microglial activation was subtle. SPECT revealed hypoperfusion throughout both hemispheres. MRS revealed a reduced N-acetyl aspartate/ creatine ratio. Conclusion: Results from this study suggested that increased DWI signals could reflect severe spongiform changes in CJD180 patients.

Copyright $\odot 2009$ S. Karger AG, Basel

\section{Introduction}

Creutzfeldt-Jakob disease (CJD) is a transmissible spongiform encephalopathy associated with the accumulation of abnormal prion protein. The disease has been classified into sporadic, familial, and infectious subtypes.

\section{KARGER}

Fax +41613061234 E-Mail karger@karger.ch www.karger.com
(C) 2009 S. Karger AG, Basel

$1420-8008 / 09 / 0286-0550 \$ 26.00 / 0$

Accessible online at:

www.karger.com/dem
Katsuya Satoh

First Department of Internal Medicine, Graduate School of Biomedical Science Nagasaki University, 1-7-1 Sakamoto

Nagasaki 852-8501 (Japan)

Tel. +8195819 7269, Fax +8195819 7270, E-Mail f1537@cc.nagasaki-u.ac.jp 
Familial CJD (fCJD) comprises approximately 15\% of all human prion disease, and is a result of point mutations or insertions in the prion protein gene (PRNP). To date, 30 subtypes of the familial form have been determined, of which 24 are due to mutations and 6 are a result of insertions. CJD, with a causative point mutation of valine to isoleucine at codon 180 (V180I) in the PRNP, is a rare type of fCJD, with only 2 cases reported from Europe $[1,2]$. However, this mutation is recognized as the most common cause of fCJD in Japan.

Jin et al. [3] reported that CJD patients with the V180I (CJD180) mutation exhibit characteristic clinical features, and that the clinical and neuroradiological findings of CJD180 patients vary from classical CJD patients in: (1) older onset age, (2) slower disease progression, (3) unique clinical symptoms, such as greater cortical dysfunction, which is less frequent in sporadic CJD (sCJD) patients, with none of the visual or cerebellar symptoms that are frequently observed in SCJD patients, (4) reduced rate of brain-specific proteins, such as neuron-specific enolase (NSE) and 14-3-3 protein, in cerebrospinal fluid (CSF) samples, and (5) the lack of a periodic sharp discharge (PSD) in the electroencephalogram (EEG) throughout the course of disease.

Diffusion-weighted imaging (DWI) may be useful in the premortem diagnosis of $\mathrm{SCJD}$, and recent reports have suggested its usefulness in fCJD cases. Jin et al. [3] reported that diffuse cortical high-intensity DWI signals are a characteristic feature of CJD180. The exact mechanisms responsible for high-intensity signals on DWI have not yet been established. Many reports have described the relationship between autopsy and DWI findings. However, brain biopsy is rarely performed, and autopsy results are not always consistent with lesions revealed by DWI abnormalities and do not always reflect the pathogenesis of DWI abnormalities. This confirms the importance of reporting and analyzing biopsy cases for neuropathology. Neuropathological findings in CJD180 patients have revealed significant spongiform changes throughout all cell layers of the gray matter, and neuronal numbers were relatively preserved, with very little abnormal prion protein expression.

A detailed analysis of the pathogenesis, which was reflected in abnormal pathological features in DWI-based neuroimaging and biochemical markers of CSF, was performed. In addition, 3 CJD180 patients were studied using MRI, magnetic resonance spectroscopy (MRS), and single photon emission-computed tomography (SPECT).

\section{Materials and Methods}

\section{Subjects}

Case 1. A 70-year-old woman was admitted with progressive memory loss. Approximately 1 year prior to admission, neighbors began noticing that the patient exhibited amnesia. Six months later, the patient began to forget things and became disoriented. Finally, the patient was unable to walk without support and was admitted to hospital. DWI demonstrated diffuse bilateral high-intensity signals in the cerebral cortex, caudate nucleus and putamen, but was predominant in the left hemisphere. The patient was right-handed. Therefore, a brain biopsy from the right frontal cortex was performed. The brain biopsy area corresponded to the region of increased DWI signals. Following CJD diagnosis, she was transferred to our university hospital. Upon admission, the patient was bedridden and exhibited myoclonic jerks and startle reactions. Muscle rigidity was present in all extremities, and deep tendon reflexes were exaggerated. CSF analysis was normal, except for negative 14-3-3 protein and elevated total tau (t-tau) protein. The patient exhibited no PSD on EEG. Genetic analysis revealed a V180I point mutation, leading to the diagnosis of fCJD. She was not able to follow simple commands and to move to the bed alone during the 12-month period after onset.

Case 2. A 67-year-old woman was admitted with a 6-month history of progressive aphasia. The past medical and family histories were unremarkable apart from cholecystectomy 17 years earlier. Upon examination, the patient was disoriented and forgetful of recent events. She did not exhibit cerebellar ataxia or myoclonus. The patient had been diagnosed with dementia of Alzheimer's type (DAT) in another hospital, but the symptoms progressed more rapidly than typical DAT. She was able to follow simple commands, but gradually lost the ability to walk.

$\mathrm{T}_{2}$-weighted imaging (T2WI) and DWI revealed increased signals in the bilateral tempoparietal cortex, predominantly in the left hemisphere. EEG revealed no PSD. Detection of the codon 180 point mutation in PRNP confirmed the diagnosis of fCJD.

The patient gradually developed speech difficulties over a 6 month period and was not able to speak by 7 months after onset. She could not follow simple commands and move to the bed alone during the 12-month period after onset.

Case 3. A 74-year-old man became disoriented while returning home from shops to which he was accustomed to traveling back and forth. The patient was unable to walk unassisted, developed urinary incontinence and began to exhibit progressive memory disturbances 1 month later. He was admitted to our hospital, and DWI abnormalities were noted. A double mutation at codons 180 and 232 (Met/Arg) of PRNP was detected, leading to a diagnosis of fCJD. The patient gradually developed speech difficulties by 4 months after onset and was unable to speak by 6 months after onset. The patient could not follow simple commands or move to the bed unassisted by 12 months after onset.

During World War II, on August 9, 1945, the city of Nagasaki was destroyed by an atomic bomb, killing tens of thousands of people. As a result of this tragedy, detailed family histories of these CJD patients remain unknown.

Social and personal conduct disorders, such as frontotemporal lobar degeneration (FTLD), and behavioral and psychological symptoms of dementia, such as DAT, were not observed in the 3 CJD180 patients. However, depressive symptoms and sleep dis- 
turbances were noted at onset. The patients did not exhibit akinetic mutism during their lifetime.

\section{Biochemical Analysis of CSF Samples}

CSF samples from all 3 patients were analyzed by ELISA for $\mathrm{t}$ tau protein, phosphorylated tau (p-tau) protein, S100 protein, NSE, and prostaglandin $\mathrm{E}_{2}\left(\mathrm{PGE}_{2}\right)$ concentrations, as well as by Western blot analysis for 14-3-3 protein expression. A polyclonal antibody specific for the $\beta$-isoform of 14-3-3 protein (sc-639; Santa Cruz, Calif., USA) was used in combination with an enhanced chemiluminescence detection kit (Amersham Buchler, Braunschweig, Germany). ELISAs were performed according to the manufacturer's instructions, using an identical standard in all experiments.

\section{Brain MRI Procedure}

MRI was performed on all subjects using a 1.5-tesla magnetic resonance unit (General Electric Medical System, Milwaukee, Wisc., USA) with $\mathrm{T}_{1}$-weighted imaging (T1WI) [repetition time $(\mathrm{TR})=400 \mathrm{~ms}$, echo time $(\mathrm{TE})=9 / \mathrm{Fr} \mathrm{ms}], \mathrm{T} 2 \mathrm{WI}(\mathrm{TR}=3,000 \mathrm{~ms}$, $\mathrm{TE}=97 \mathrm{~ms})$, fluid attenuation inversion recovery $(\mathrm{TR}=8,002 \mathrm{~ms}$, $\mathrm{TE}=104 / \mathrm{ms}$ ), and DWI sequences of $5-\mathrm{mm}$ slice thickness. The acquired data were analyzed using Digital Imaging and Communications in Medicine (DICOM) format.

SPECT Image Analysis Using eZIS and N-Isopropyl-p-[123I]Iodoamphetamine

SPECT was performed using ${ }^{99 \mathrm{~m}} \mathrm{Tc}$-ethyl cysteinate dimer ( $\left.{ }^{99 \mathrm{~m}} \mathrm{Tc}-\mathrm{ECD}\right)$ and $\mathrm{N}$-isopropyl- $p-\left[{ }^{123} \mathrm{I}\right]$-iodoamphetamine $\left({ }^{123} \mathrm{I}\right.$ IMP) as a tracer in all subjects. Obtained images were anatomically standardized with an original ${ }^{99 \mathrm{~m}}$ Tc-ECD template using the easy $Z$-score imaging system (eZIS) established by Matsuda et al. [4].

\section{Magnetic Resonance Spectroscopic Analysis}

Single-voxel ${ }^{1} \mathrm{H}$-MRS was performed. Spectra were acquired from an 8 - $\mathrm{ml}$ cubic volume of interest centered on the right cerebral cortex.

\section{Neuropathological Investigation}

The right frontal lobe brain biopsy from case 1 corresponded with increased DWI signals. The time interval between brain biopsy and DWI was 1 day.

Formalin-fixed, paraffin-embedded sections of brain biopsy tissue were subjected to histological analyses, including hematoxylin-eosin (HE) staining and $\operatorname{PrP}^{\mathrm{Sc}}$ immunohistochemistry with 3F4 monoclonal antibody. Glial activation (astrocytic gliosis and microglia activation) was assessed by immunohistochemistry on tissue sections using S100 protein and CD68. The neuropathological findings (HE staining, $\mathrm{S} 100$ protein, $\mathrm{CD} 68$, and 3F4 immunohistochemistry) were assessed in biopsy tissue from case 1 and compared with autopsy tissue from 4 typical sCJD cases.

\section{Statistical Analysis}

Comparisons of clinical symptoms between the present cases, 7 cases described in previous reports, and pooled data from sCJD patients were performed using the $\chi^{2}$ test and Student's t test. The Medical Ethics Committee of Nagasaki University School of Medicine approved this study, and the participants provided written informed consent.

\section{Results}

\section{Clinical Findings in CJD180 Patients}

Clinical features from the 3 cases are summarized in table 1.

The age at onset was $69 \pm 1.41$ years in the 3 CJD180 patients. When the 4 CJD180 patients described in previous reports were combined with this analysis, the age at onset of the 7 CJD180 patients was $70.3 \pm 3.9$ years. There was no statistically significant difference in age at onset between the present CJD180 patients and the previously described sCJD patients $(65.3 \pm 11.6$ years old; $\mathrm{n}=$ 128).

Appearance of myoclonic jerk from disease onset was $5.33 \pm 0.93$ months in the present 3 CJD180 patients, and $8.0 \pm 4.5$ months in all 7 CJD180 patients (including 4 previously described patients). Myoclonic jerk was identified in all cases, but the duration to appearance was longer in CJD180 patients compared with sCJD patients (2.7 \pm 2.4 months). The myoclonic jerks were less remarkable in the present patients compared with the sCJD patients, and the myoclonic jerks of all CJD180 patients exhibited a similar frequency to Parkinson's disease tremors (5$9 \mathrm{~Hz})$.

Time to appearance of akinetic mutism from disease onset was $12.3 \pm 4.50$ months in the present 3 CJD180 patients and $11.7 \pm 3.34$ months in all 7 CJD180 patients (including 4 previously described patients). The time to appearance of akinetic mutism was longer in CJD180 patients compared with $\mathrm{sCJD}$ patients $(\mathrm{p}<0.01)$. Survival time of the present 3 CJD180 patients was $30.3 \pm 4.78$ months.

\section{Biochemical Analysis of CSF}

The biochemical markers used for CSF analysis are listed in table 1.

CSF from all patients was negative for 14-3-3 protein (present and previously described cases). 14-3-3 protein was detected in $87.7 \%$ of the sCJD patients. Therefore, 14-3-3 protein expression in CSF was not used as a diagnostic marker for CJD180.

In the patients from this study, t-tau protein titers were greater than the cut-off level $(1,300 \mathrm{pg} / \mathrm{ml})$, but much less than in the sCJD cases $(5,689 \pm 169 \mathrm{pg} / \mathrm{ml} ; \mathrm{n}=128$; data not shown). NSE concentrations in all 3 cases were less than the cut-off value $(35 \mathrm{ng} / \mathrm{ml})$, but greater than the concentrations in individuals with neurodegenerative disorders (10.35 $\pm 4.35 \mathrm{ng} / \mathrm{nl} ; \mathrm{n}=100)$. S100 protein and $\mathrm{PGE}_{2}$ protein concentrations were below the detection thresholds. 
Table 1. Profiles of the 3 cases with CJD180

\begin{tabular}{|c|c|c|c|c|c|}
\hline & \multicolumn{3}{|l|}{ Our cases } & \multicolumn{2}{|l|}{ Previously reported ${ }^{1}$} \\
\hline & case 1 & case 2 & case 3 & CJD180 & sCJD \\
\hline Age/sex & $70 / \mathrm{F}$ & $67 / \mathrm{F}$ & $70 / \mathrm{M}$ & $70.3 \pm 3.9$ & $65.3 \pm 11.6$ \\
\hline Family history & - & - & - & - & - \\
\hline Myoclonic jerk, months & 6 & 6 & 4 & $8.0 \pm 4.5$ & $2.7 \pm 2.4$ \\
\hline Visual symptoms & - & - & - & $0 \%(0 / 4)$ & $24 \%(23 / 96)$ \\
\hline Cerebellar symptoms & - & - & - & $0 \%(0 / 4)$ & $12.5 \%(12 / 96)$ \\
\hline Akinetic mutism, months & 12 & 18 & 7 & $11.3 \pm 2.2$ & $3.5 \pm 2.8$ \\
\hline Total tau, pg/ml & 3,811 & 2,325 & 3,675 & $\mathrm{NE}$ & $89.6 \%(86 / 96)$ \\
\hline Phosphorylated tau, pg/ml & 39.4 & 40.8 & 36.1 & $\mathrm{NE}$ & $\mathrm{NE}$ \\
\hline NSE, ng/ml & 34 & 22 & 13 & $50 \%(2 / 4)$ & $72.7 \%(48 / 66)$ \\
\hline $\mathrm{S} 100$ protein, $\mathrm{ng} / \mathrm{ml}$ & 0.23 & 0.35 & 0.13 & $\mathrm{NE}$ & $\mathrm{NE}$ \\
\hline $14-3-3$ protein & - & - & - & $0 \%$ & $87.7 \%(57 / 65)$ \\
\hline PSD & - & - & - & $0 \%$ & $94 \%(110 / 116)$ \\
\hline Codon 129 in PRNP & $\mathrm{M} / \mathrm{M}$ & $\mathrm{M} / \mathrm{M}$ & $\mathrm{M} / \mathrm{M}$ & MM 40\%; MV60\% & MM 91\%; MV 9\% \\
\hline Codon 219 in PRNP & $\mathrm{E} / \mathrm{E}$ & $\mathrm{E} / \mathrm{E}$ & $\mathrm{E} / \mathrm{E}$ & E/E $100 \%$ & E/E 92\%; E/K8\% \\
\hline Type of $\operatorname{PrP}^{S c}$ & type $1+2$ & $\mathrm{NE}$ & $\mathrm{NE}$ & $\mathrm{NE}$ & type 1 or type 2 \\
\hline
\end{tabular}

Range of t-tau protein levels: $800-15,000 \mathrm{ng} / \mathrm{ml}, \mathrm{CJD}$ with values $>1,300 \mathrm{pg} / \mathrm{ml}$.

Range of p-tau protein levels: 8-120 ng/ml.

Range of NSE levels: $2-200 \mathrm{ng} / \mathrm{ml}$, CJD with values $>35 \mathrm{ng} / \mathrm{ml}$.

Range of S100 protein levels: $0.001-25 \mathrm{ng} / \mathrm{ml}, \mathrm{CJD}$ with values $>2.2 \mathrm{ng} / \mathrm{ml}$.

We analyzed the cutoff data of t-tau protein, NSE and S100 protein of CSF among 128 CJD patients and 100 non-CJD patients (DAT, vascular dementia, Pick's disease, Parkinson's disease, corticobasal degeneration, Huntington's disease, frontotemporal dementia, progressive supranuclear palsy, mild cognitive impairment, amyotrophic lateral sclerosis, temporal epilepsy, limbic encephalopathy, paraneoplastic cerebellar degeneration/Lambert-Eaton myasthenic syndrome, MELAS and encephalopathy due to unknown etiology). The most appropriate cutoff levels of biomarkers (t-tau protein, NSE and S100 protein of CSF) in CJD patients were evaluated using the receiver-operating characteristics curve method. The sensitivities for t-tau protein, 14-3-3 protein, NSE and S100 protein of CSF in classical CJD patients $(\mathrm{n}=128)$ were $95.9,88.7,81.5$ and $33.1 \%$, respectively (data not shown).

In all 3 patients, the polymorphism in PRNP at codon 129 was homozygous for methionine (M/M), while the polymorphism in PRNP at codon 219 was homozygous for glutamic acid (E/E). $\mathrm{NE}=$ Not examined; $\mathrm{M}=$ methionine; $\mathrm{V}=$ valine; $\mathrm{K}=$ lysine; $\mathrm{E}=$ glutamic acid

${ }^{1}$ As previously reported by Jin et al. [3], the average level of $t-$ tau protein of classical CJD patients $(n=128)$ was $5,689 \pm 169$ $\mathrm{pg} / \mathrm{ml}$ (average $\pm 1 \mathrm{SD}$ ).

\section{Neuroimaging (MRI, MRS, SPECT)}

According to the MRI results, all cases demonstrated a wide range of cerebral cortical ribbons, which were depicted as low-intensity areas in the bilateral putamen, caudate head, and cerebral cortex by T1WI, and as highintensity areas by T2WI, fluid attenuation inversion recovery, and DWI. The cortical lesion was not always symmetric (cases 1 and 2). Basal ganglia lesions were detected, and the caudate head was detected in all cases (fig. 1). The cerebellum and brain stem were not abnormal.

MRS revealed decreased $\mathrm{N}$-acetyl aspartate (NAA)/ creatine $(\mathrm{Cr})$ and choline $(\mathrm{Cho}) / \mathrm{Cr}$ ratios in the cerebral cortices of all patients (table 2), whereas there were no changes in myoinositol (MI) levels (fig. 2). SPECT images from case 2 revealed a widespread decreased perfusion in both cerebral hemispheres, in particular the left temporal cortex (table 2). Highly insensitive DWI regions were observed throughout the cerebral cortex in the CJD180 cases and were similar to the smaller volume area observed on SPECT (fig. 2). Regional cerebral blood flow in the cerebral cortex was less in the CJD180 cases than in the sCJD cases.

\section{Diagnosis of CJD}

The characteristic clinical finding in the 3 CJD180 patients was progressive dementia and memory disturbance; the time course of the progressive dementia in the 3 CJD180 patients was different from that of DAT or FTLD, and was also different from typical sCJD patients. The psychiatric findings in the 3 CJD180 patients includ- 


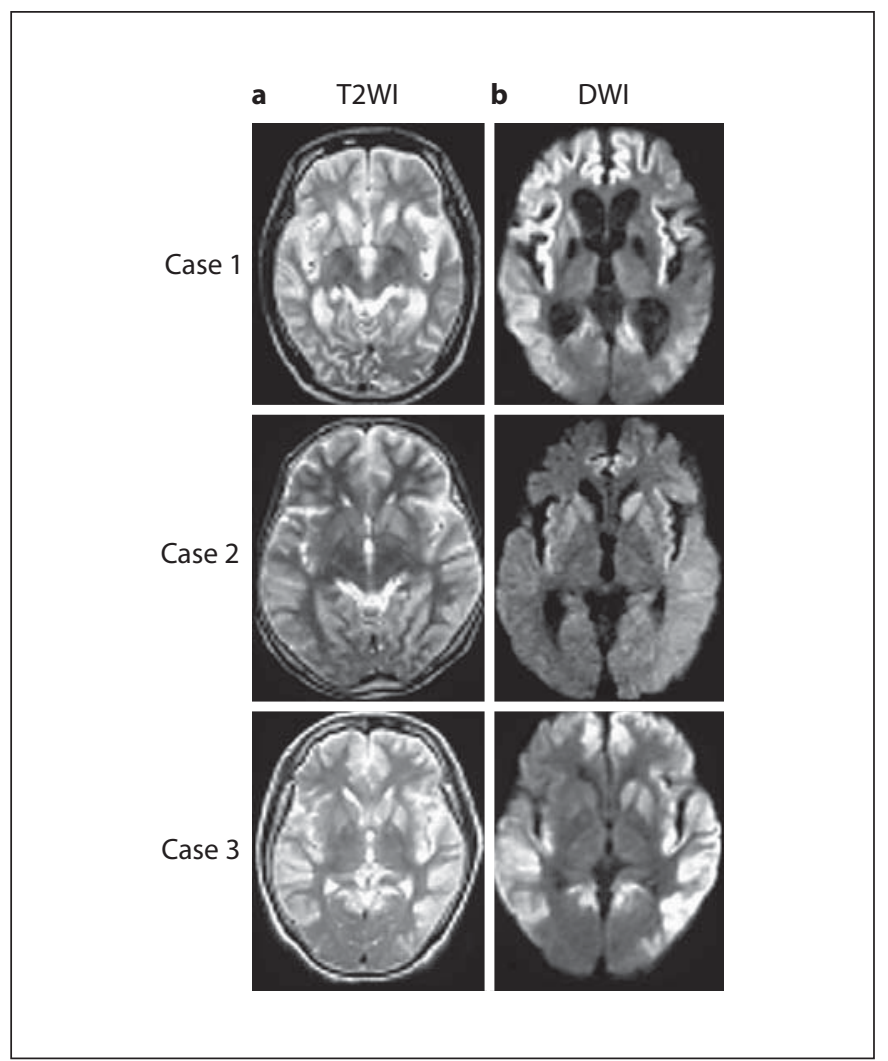

Fig. 1. Axial $\mathrm{T}_{2^{-}}$(a) and diffusion-weighted (b) MR images from the 3 CJD cases with the V180I mutation, revealing extensive cortical hyperintensity lesions. Hyperintense signals in the bilateral caudate nucleus and putamen are also demonstrated, but these are subtle compared with the cortical lesions. Medial regions of the occipital lobes are not involved.

Table 2. MRS in the 3 cases with CJD180 and 4 cases with sCJD

\begin{tabular}{lcccc}
\hline & Case 1 & Case 2 & Case 3 & sCJD $(\mathrm{n}=4)$ \\
\hline NAA/Cr & 0.79 & 1.1 & 1.3 & $1.83 \pm 0.20$ \\
Cho/Cr & 1.18 & 1.02 & 0.91 & $1.49 \pm 0.20$ \\
MI & 30 & 28 & 11 & $<30$
\end{tabular}

sCJD $(n=4)$ patients were definite cases and the molecular type of the abnormal prion protein in SCJD cases was type 2 on Parchi's classification [6].

ed depression and sleep disturbance, but disinhibition and asocial behavior were not determined in FTLD of the 3 CJD180 cases. In addition, behavioral and psychological symptoms of dementia in DAT were not recognized in the 3 CJD180 cases. a

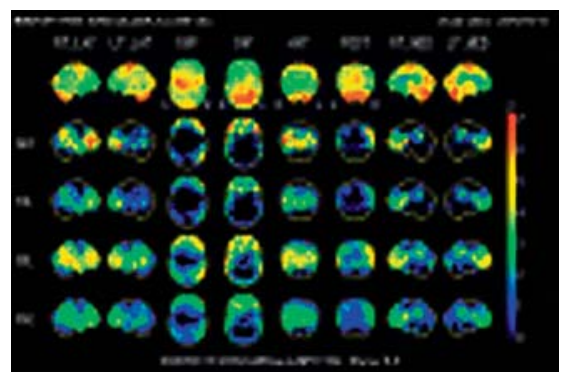

b

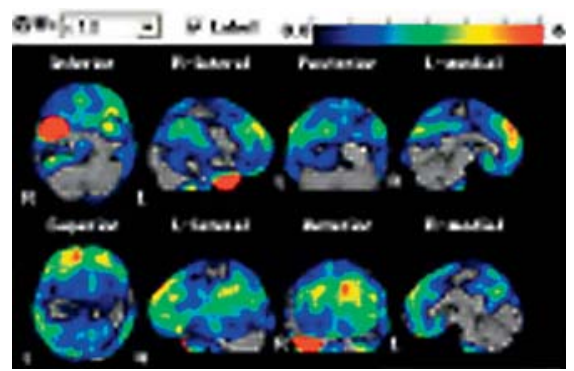

c

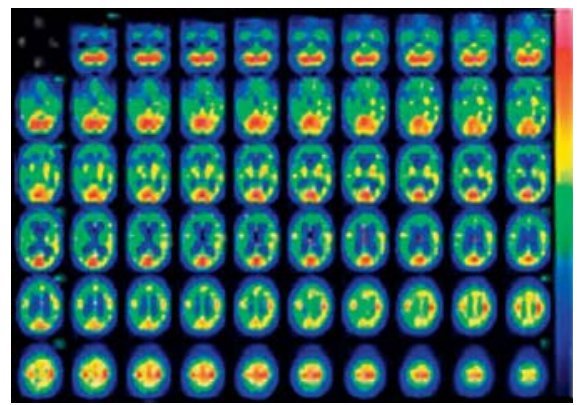

Fig. 2. SPECT analysis in case 1 (CJD180). a ${ }^{123}$ I-IMP SPECT demonstrated decreased uptake. b eZIS analysis in SPECT performed using ${ }^{99 \mathrm{~m}} \mathrm{Tc}$-ECD. c 3DSRT analysis in SPECT performed using ${ }^{99 \mathrm{~m}} \mathrm{Tc}-\mathrm{ECD}$.

The 3 CJD180 patients were misdiagnosed with DAT. CSF samples were analyzed from 100 patients with various neurodegenerative disorders using Western blot to detect 14-3-3 protein, $\mathrm{t}$-tau protein, and $\mathrm{p}$-tau protein expressions. t-tau protein concentration in CSF of CJD patients was $>1,200 \mathrm{pg} / \mathrm{ml}$. However, the level of $\mathrm{t}$-tau protein in CSF of neurodegenerative disorder patients was $200-500 \mathrm{pg} / \mathrm{ml}$, and the level of $\mathrm{t}$-tau protein from CSF of DAT patients was $400-1,400 \mathrm{pg} / \mathrm{ml}$. Elevated t-tau protein was also detected in 3 patients from the non-CJD group. Elevated t-tau protein levels were observed in 2 


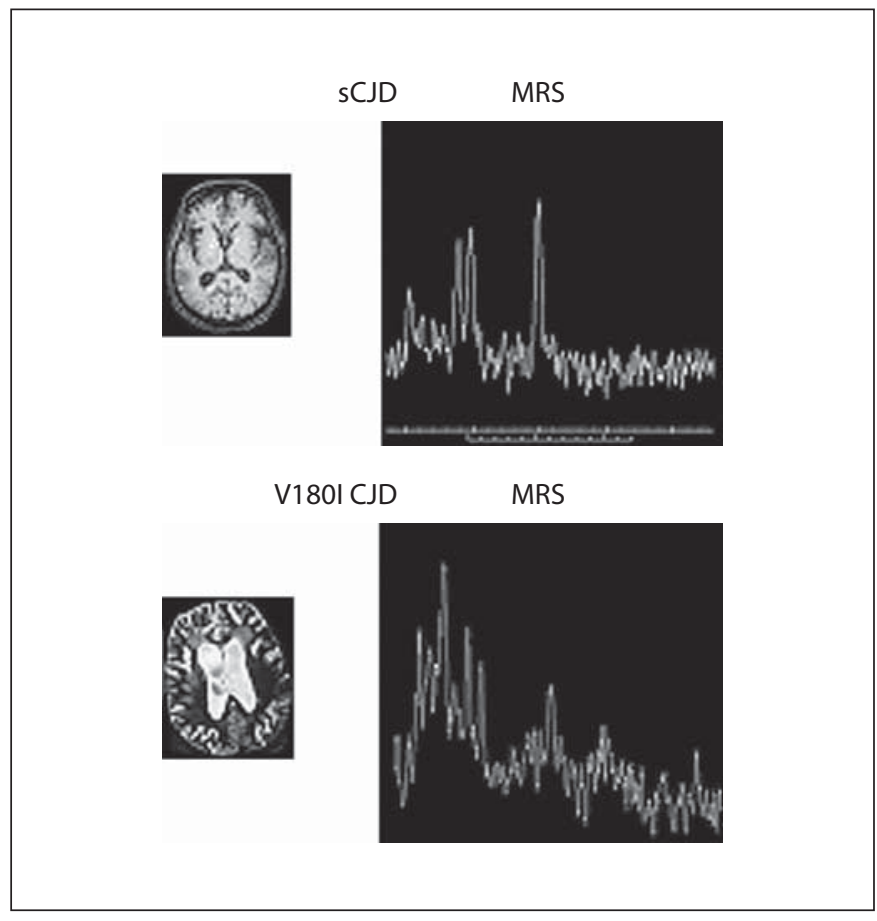

Fig. 3. ${ }^{1} \mathrm{H}$-MRS in case 1 exhibits decreased cortical gray matter NAA/Cr.

patients with DAT and in 1 patient with cerebrovascular disease in the acute phase. To distinguish CJD patients from non-CJD patients with elevated $t$-tau protein in CSF, the ratio of $\mathrm{p}$-tau and $\mathrm{t}$-tau proteins was compared. The $\mathrm{p}$-/t-tau ratio was significantly greater in DAT patients compared with CJD patients. Some diseases revealed $>2,000 \mathrm{pg} / \mathrm{ml}$ t-tau protein in the CSF of CJD patients. When it was difficult to distinguish CJD from DAT patients, the ratio of $\mathrm{p}$-tau and $\mathrm{t}$-tau proteins was compared.

The highest level of t-tau protein in the FTLD patients was $670 \mathrm{pg} / \mathrm{ml}$, and there were no FTLD patients with CSF values $>1,000 \mathrm{pg} / \mathrm{ml}$ t-tau protein, which was consistent with previous reports [5]. Results from CSF analysis, clinical disease course, and the neuroradiological findings in the V180I CJD patients varied between the DAT and FTLD patients.

\section{Neuropathology}

HE staining revealed severe spongiform changes in the cerebral cortex, which were more moderate in the CJD180 patients compared with the sCJD cases. The CJD180 patients exhibited a relatively limited neuronal loss compared with the sCJD patients (fig. 4a).

DWI and Brain Biopsy of Familial CJD (V180I)

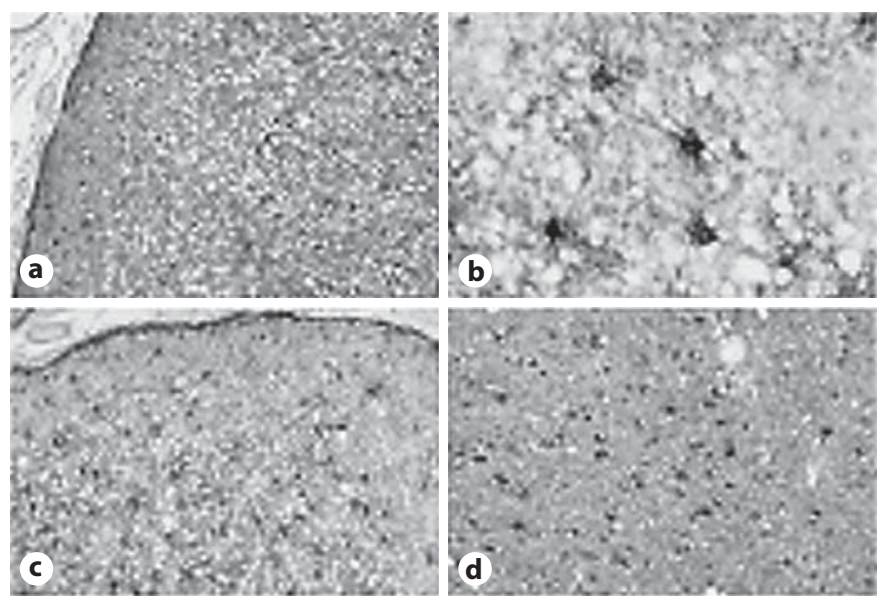

Fig. 4. Pathological findings in the right frontal lobe of case 1 reveal severe spongiform changes and neuronal loss. However, astrocytic gliosis, accumulation of $\mathrm{PrP}^{\mathrm{Sc}}$, and microglial activation are less apparent in the cerebral cortex. a HE staining. b CD68 staining. c S100b protein staining $(\times 10)$. d S100b protein staining $(\times 200)$.

Immunostaining with 3F4 antibody was not detected in 1 patient (case 1), but all control cases of sCJD expressed 3F4 protein. CD68 expression was positive, but the number of CD68-positive microglia in the present sCJD cases was greater than in the CJD180 patients (fig. 4b). S100 immunohistochemistry demonstrated mild astrocytic gliosis in the CJD180 patients. However, 2 sCJD (early stage and middle stage) and 2 late-stage patients exhibited severe and moderate astrocytic gliosis, respectively (fig. $4 \mathrm{c}, \mathrm{d}$ ).

\section{Typing of Protease-Resistant Prion Protein}

According to Western blot analysis, the abnormal prion protein in the CJD180 cases was type 2 according to Parchi's classification [6].

\section{Discussion}

Neuropathological examination of tissue biopsies from case 1 demonstrated an unusual presentation compared with the biopsy sections from sCJD cases. First, all cell layers of the gray matter exhibited marked spongiform changes. Second, neuronal numbers were relatively preserved. Third, no abnormal prion protein expression was detected. Fourth, microglial activation was subtle. These results were consistent with the unique characterizations from previous reports [7-9].

Dement Geriatr Cogn Disord 2009;28:550-557 
Table 3. Neuropathological findings in 1 case with CJD180 (case 1) and 4 cases with $\mathrm{SCJD}$

\begin{tabular}{llllll}
\hline & $\begin{array}{l}\text { CJD180 } \\
\text { case 1 }\end{array}$ & $\begin{array}{lllll}\text { cCJD } \\
\text { case 1 }\end{array}$ & $\begin{array}{l}\text { sCJD } \\
\text { case 2 }\end{array}$ & $\begin{array}{l}\text { sCJD } \\
\text { case 3 }\end{array}$ & $\begin{array}{l}\text { sCJD } \\
\text { case 4 }\end{array}$ \\
\hline Neuronal loss & + & + & + & +++ & +++ \\
Astrocytic gliosis & + & ++ & ++ & + & + \\
Spongiform change & +++ & ++ & ++ & + & + \\
Microglia activation & + & +++ & ++ & ++ & ++ \\
PrP deposition & - & ++ & ++ & +++ & ++ \\
\hline
\end{tabular}

Spongiform change and neuronal loss are described as absent $(-)$, mild $(+)$, moderate $(++)$, and severe $(+++)$ on HE sections.

Astrocytosis is described as absent (-), mild (+), moderate (++), and severe $(+++)$ on sections labeled immunohistochemically with an anti-S100 antibody (polyclonal, DAKO, Glostrup, Denmark) or anti-prion protein antibody (monoclonal, clone 3F4, Senetek, Maryland Heights, Mo., USA).

Degree of prion protein deposition is described as absent $(-)$, mild $(+)$, moderate $(++)$, and strong $(+++)$ on sections labeled immunohistochemically with an anti-prion protein antibody (monoclonal, clone 3F4, DAKO, Japan).

Microglia activation (the number of CD68-positive microglia) is described as follows: no staining $(-)$, slightly staining $(+)$, moderate staining $(++)$, and strong staining $(+++)$.

More than 30 CJD patients have undergone brain biopsies, but only 3 reports [10-12] discussed the correlation between neuropathological and DWI findings in brain biopsy tissue. In other studies, brain biopsies were used only as a diagnostic method to rule out progressive dementia.

Heinemann et al. [10] suggested that the correlation between DWI and neuropathological findings in brain biopsies could be a result of neuronal loss and spongiform changes. Spongiform neuronal degeneration was demonstrated to underlie the increased DWI signals in sCJD patients as shown by Kim et al. [11]. In addition, See et al. [12] suggested that fluid accumulation within cytoplasmic vacuoles of the neuropil, or astrogliosis, contributes to a restricted diffusion range that underlies DWI abnormalities. Microglial activation has been suggested as another possible cause of DWI abnormalities. A relationship between DWI abnormalities and accumulation of $\mathrm{PrP}^{\mathrm{Sc}}$ has been suggested as a possible causative mechanism $[6,13]$. Results from the present study demonstrated very low abnormal prion protein expression. These results suggest that there is not a strong relationship between DWI abnormalities and $\operatorname{PrP}^{\mathrm{Sc}}$ accumulation.
It is difficult to determine the mechanisms underlying DWI abnormalities based on results from a single brain biopsy. Although the present study utilized single brain biopsies, pathological analyses clearly revealed pathological findings in the SCJD cases (table 3). Nevertheless, the mechanism underlying the DWI abnormalities was assumed to be a result of severe spongiform changes as revealed by neuropathological findings.

Manners et al. [14] reported a DWI and $\mathrm{T}_{2}$-weighted echoplanar MRI study comprising $10 \mathrm{sCJD}$ patients. At postmortem, the sCJD patients were evaluated for semiquantitative assessment of gliosis and neuronal loss, spongiform changes, and abnormal $\mathrm{PrP}$ protein deposition in 4 cortical regions (occipital, parietal, temporal cortex and cingulate gyrus), the thalamus and the striatum, for a total of 60 regions of interest. Results suggested that antemortem reductions in the apparent diffusion coefficient values, typically observed in patients with sCJD, correlate with spongiform changes observed at autopsy. This was clearly established in the striatum and thalamus of the present sCJD patients, in which the extent of spongiform change did not significantly correlate with gliosis or neuronal loss. The study by Manners et al. [14] was based on extremely detailed examination and referred to one of our studies. However, the pathological characteristics of CJD180 differed in our study compared with sCJD. The pathological characteristics of CJD180 included greater spongiform changes, as well as less deposition of abnormal prion protein and astrocytic gliosis compared with sCJD.

The greatest difference between the study by Manners et al. [14] and ours is the concept. In our study, DWI signals from CJD patients were high or low during the clinical course of disease, and these signal changes were detectable.

Due to ethical considerations, brain biopsies were not performed in all cases in the present study. Therefore, the present hypothesis was addressed by supplementary biochemical analysis of CSF and neuroimaging.

Several reports have described the MRS results in CJD patients $[15,16]$. NAA is produced exclusively in neuronal mitochondria and reduced levels of NAA are considered to reflect neuronal loss or dysfunction. In turn, Cho levels reflect membrane synthesis and graduation. MI is thought to be located only in glial cells and is, therefore, considered to be a glial marker. A previous report described that reduced levels of NAA correlate with histological neuronal loss and astrocytic gliosis in CJD patients. Some studies have reported reduced NAA levels as a feature of sCJD followed by spongiform changes accom- 
panying neuronal loss and gliosis. Although these studies have reported increased levels of MI in sCJD patients, results from the present study demonstrated reduced NAA/ $\mathrm{Cr}$ and $\mathrm{Cho} / \mathrm{Cr}$ ratios but normal MI levels in the right frontal lobe. These results were assumed to reflect the severe spongiform change and neuronal loss as revealed by MRS.

SPECT imaging revealed marked cerebral blood flow reduction, predominantly in cerebral cortical regions corresponding to brain areas with high-intensity DWI signals. This was most likely due to severe neuronal loss and/or severe spongiform change. However, one study reported a preserved cerebral blood flow, or increased perfusion, and marked high-intensity DWI changes [17]. Autopsy findings revealed mild neuronal loss, which most likely was responsible for the preserved perfusion. Moreover, SPECT results revealed hypoperfusion due to spongiform change and mild neuronal loss.

CSF S100 protein levels are affected by astrocytic gliosis, and CSF $\mathrm{PGE}_{2}$ levels are influenced by microglial activation. The present results clearly identified reduced levels of S100 in CSF and decreased $\mathrm{PGE}_{2}$ titers. CSF t-tau protein concentration was increased due to neuronal loss, resulting in lower t-tau protein levels in CJD180 patients compared with classical CJD patients. One of the neuropathological features in SCJD patients is severe neuronal loss in the cerebral cortex. Accordingly, neuropathological findings and CSF analysis ( $\mathrm{t}$-tau protein) revealed that neuronal loss in CJD180 patients was less than in sCJD patients. Biochemical CSF markers do not necessarily reflect the clinical condition, but CSF biomarker levels can be used as a pathological index. CSF analysis from the present study demonstrated mild astrocytic gliosis or spongiform change in the CJD180 patients.

Biochemical CSF analysis and neuroimaging results suggested that the cause of abnormal DWI signals was spongiform change and neuronal loss. However, this is difficult to prove. Indeed, results demonstrated that neurons in CJD180 patients were better preserved than in sCJD patients, and the spongiform change in CJD180 patients was more moderate than in the 4 typical sCJD cases examined.

In conclusion, results from the present study suggested that high-intensity DWI signals in CJD180 patients were influenced by spongiform changes.

\section{References}

1 Genetic prion disease: the EUROCJD experience. Hum Genet 2005;118:166-174.

-2 Chasseigneaux S, Haik S, Laffont-Proust I, et al: V180I mutation of the prion protein gene associated with atypical $\mathrm{PrP}^{\mathrm{Sc}}$ glycosylation. Neurosci Lett 2006;408:165-169.

- 3 Jin K, Shiga Y, Shibuya S, et al: Clinical features of Creutzfeldt-Jakob disease with V180I mutation. Neurology 2004;62:502-505.

-4 Matsuda H, Mizumura S, Nagao T, et al: An easy Z-score imaging system for discrimination between very early Alzheimer's disease and controls using brain perfusion SPECT in a multicentre study. Nucl Med Commun 2007:28:199-205.

5 Satoh K, Shirabe S, Eguchi H, et al: 14-3-3 protein, total tau and phosphorylated tau in cerebrospinal fluid of patients with Creutzfeldt-Jakob disease and neurodegenerative disease in Japan. Cell Mol Neurobiol 2006; 26:45-52.

-6 Parchi P, Castellani R, Capellari S, et al: Molecular basis of phenotypic variability in sporadic Creutzfeldt-Jakob disease. Ann Neurol 1996;39:767-778
7 Iwasaki Y, Sone M, Kato T, et al: Clinicopathological characteristics of Creutzfeldt-Jakob disease with a PrP V180I mutation and M129V polymorphism on different alleles (in Japanese). Rinsho Shinkeigaku 1999;39: 800-806.

-8 Matsumura T, Kojima S, Kuroiwa Y, et al: An autopsy-verified case of Creutzfeldt-Jakob disease with codon 129 polymorphism and codon 180 point mutation (in Japanese). Rinsho Shinkeigaku 1995;35:282-285.

$\checkmark 9$ Shindo K, Shimosaka C, Ohta E, Inada H, Togashi S, Nitta K, Nagasaka T, Kato R, Kobayashi M, Shiozawa Z: Autopsy-proven Creutzfeldt-Jakob disease with a codon 180 mutation showing dissociation between diffusion-weighted magnetic resonance imaging and single-photon emission computed tomography findings. Is this a suggestive finding in long survival? Eur Neurol 2006; $56: 46-49$.

10 Heinemann U, Krasnianski A, Meissner B, et al: Brain biopsy in patients with suspected Creutzfeldt-Jakob disease. J Neurosurg 2008; 109:735-741.

$\checkmark 11$ Kim HC, Chang KH, Song IC, et al: Diffusion-weighted MR imaging in biopsy-proven Creutzfeldt-Jakob disease. Korean J Radiol 2001;2:192-196.
12 See SI, Pan A, Seah A, et al: Case reports of two biopsy-proven patients with CreutzfeldtJakob disease in Singapore. Ann Acad Med Singapore 2004;33:651-655.

13 Haik S, Dormont D, Faucheux BA, et al: Prion protein deposits match magnetic resonance imaging signal abnormalities in Creutzfeldt-Jakob disease. Ann Neurol 2002; 51:797-799.

14 Manners DN, Parchi P, Tonon C, et al: Pathologic correlates of diffusion MRI changes in Creutzfeldt-Jakob disease. Neurology 2009; 72:1425-1431.

15 Pandya HG, Coley SC, Wilkinson ID, Griffiths PD: Magnetic resonance spectroscopic abnormalities in sporadic and variant Creutzfeldt-Jakob disease. Clin Radiol 2003; 58:148-153

16 Lim CC, Tan K, Verma KK, Yin H, Venketasubramanian $\mathrm{N}$ : Combined diffusionweighted and spectroscopic MR imaging in Creutzfeldt-Jakob disease. Magn Reson Imaging 2004;22:625-629.

17 Sunada I, Ishida T, Sakamoto S, Tsuyuguchi $\mathrm{N}$ : A discrepancy between Tc-99m HMPAO SPECT and Tc-99m ECD SPECT in Creutzfeldt-Jacob disease. J Clin Neurosci 2004;11:648-650. 